\title{
Rerista A regentina de

\section{Efecto de los antimaláricos sobre los diferentes dominios del índice de daño SLICC en pacientes de la cohorte GLADEL}

\begin{abstract}
Guillermo J. Pons-Estel ${ }^{1,2}$, Rosana Quintana ${ }^{1,2}$, Daniel Wojdyla ${ }^{3}$, Graciela S. Alarcón ${ }^{4}$, Rosa María Serrano ${ }^{1}$, Manuel Ugarte-Gil ${ }^{5,6}$, Víctor Pimentel-Quiroz ${ }^{6}$, Enrique R. Soriano, Luis J. Catoggio7, Marina Scolnik ${ }^{7}$, Mónica Sacnun ${ }^{2}$, Verónica Saurit ${ }^{8}$, Francisco Caeiro ${ }^{8}$, Alejandro Alvarellos ${ }^{8}$, Judith Sarano ${ }^{9}$, Mercedes García ${ }^{10}$, Laura Onetti ${ }^{11}$, Cristina Drenkard ${ }^{12}$, Guillermo Berbotto ${ }^{13}$, Hugo R. Scherbarth ${ }^{14}$, Emilia Sato ${ }^{15}$, Eloisa Bonfa ${ }^{16}$, Eduardo Ferreira Borba ${ }^{16}$, Lilian Costallat ${ }^{17}$, Ricardo Xavier ${ }^{18}$, Joao C. Tavares Brenol ${ }^{18}$, Nilzio A. Da Silva ${ }^{19}$, Fernando Cavalcanti ${ }^{20}$, Loreto Massardo ${ }^{21}$, Sergio Jacobelli ${ }^{21}$, Oscar Neira ${ }^{22}$, José $F$ Molina $^{23}$, Gloria Vásquez ${ }^{24}$, José A. Gómez-Puerta ${ }^{24}$, Luis Alonso Gonzalez ${ }^{24}$, Antonio Iglesias Gamarra ${ }^{25}$, Marlene Guibert-Toledano ${ }^{26}$, Gil A. Reyes ${ }^{26}$, Mario H. Cardiel ${ }^{27}$, Virginia Pascual-Ramos ${ }^{28}$, Ignacio García de la Torre ${ }^{29}$, Leonor Barile ${ }^{30}$, Luis H. Silveira ${ }^{31}$, Mary-Carmen Amigo ${ }^{32}$, María Josefina Sauza del Pozo ${ }^{33}$, Eduardo M. Acevedo-Vásquez ${ }^{6}$, José Alfaro-Lozano ${ }^{6}$, María Inés Segami ${ }^{34}$, Rosa Chacón-Díaz ${ }^{35}$, Isaac Abadi ${ }^{35}$, María H Esteva Spinetti ${ }^{36}$, y Bernardo A. Pons-Estel ${ }^{1}$
\end{abstract}

Centro Regional de Enfermedades Autoinmunes y Reumáticas (GO-CREAR). Rosario, Argentina1; Hospital Provincial de Rosario, Rosario, Argentina²; Consultor de GLADEL'; Universidad de Alabama (Birmingham, EE.UU.) ${ }^{4}$; Hospital Nacional Guillermo Almenara Irigoyen, ESSALUD, Lima, Perús; Universidad Científica del Sur. Lima, Perú6; Sección de Reumatología, Servicio de Clínica Médica; Instituto Universitario, Escuela de Medicina, y Fundación Dr. Pedro M. Catoggio para el Progreso de la Reumatología, Hospital Italiano de Buenos Aires. Ciudad Autónoma de Buenos Aires, Argentina ; Hospital Privado, Centro Médico de Córdoba, Córdoba, Argentina ${ }^{8}$; Servicio de Inmunología, Instituto de Investigaciones Médicas "Alfredo Lanari", Ciudad Autónoma de Buenos Aires, Argentina9; Hospital Interzonal General de Agudos General San Martín, La Plata, Argentina ${ }^{10}$; Servicio de Reumatología, UHMI1, Hospital Nacional de Clínicas, Córdoba, Argentina ${ }^{11}$; Department of Medicine, Division of Rheumatology, Emory School of Medicine. Atlanta, Georgia, USA ${ }^{12}$; Hospital Escuela Eva Perón, Granadero Baigorria, Argentina ${ }^{13}$; Unidad de Reumatología y Enfermedades Autoinmunes Sistémicas, HIGA Dr. Oscar Alende. Mar del Plata, Argentina ${ }^{14}$; Universidade Federal da Sao Paulo (UNIFESP), Sao Paulo, Brasil ${ }^{15}$; Universidade da Sao Paulo, Sao Paulo, Brasil ${ }^{16}$; Universidade Estadual da Campinas, Campinas, Brasil ${ }^{17}$; Hospital da Clinicas da Porto Alegre, Universidade Federal do Rio Grande do Sul, Brasil ${ }^{18}$; Faculdade de Medicina, Universidade Federal de Goias, Goiania, Brasil ${ }^{19}$; Universidade Federal da Pernambuco, Pernambuco, Brasil20; Facultad de Medicina. Universidad San Sebastián, Santiago, Chile ${ }^{21}$; Hospital del Salvador, Facultad de Medicina, Universidad de Chile, Santiago, Chile ${ }^{22}$; Centro Integral de Reumatología, Reumalab. Medellín, Colombia ${ }^{23}$; Grupo de Inmunología Celular e Inmunogenética, Grupo de Reumatología, Facultad de Medicina, Universidad de Antioquia. Medellín, Colombia24; Servicio de Reumatología, Hospital Clinic. Barcelona, España ${ }^{25}$; Centro de Investigaciones Médico Quirúrgicas (CIMEQ), La Habana, Cuba ${ }^{26}$; Centro de Investigación Clínica de Morelia SC, Morelia, México ${ }^{27}$; Departamento de Inmunología y Reumatología, Instituto Nacional de Ciencias Médicas y Nutrición Salvador Zubirán, México DF, México28; Hospital General de Occidente de la Secretaría de Salud, Guadalajara, México29; Ángeles del Pedregal, Ciudad de México, México30; Departamento de Reumatología, Instituto Nacional de Cardiología "Ignacio Chávez". Ciudad de México, México ${ }^{31}$; Centro Médico ABC, Ciudad de México, México ${ }^{32}$; Servicio de Reumatología, Instituto Mexicano de Seguro Social, Hospital de Especialidades No 25, Monterrey, NL, Mexico33; Hospital Nacional Edgardo Rebagliatti Martins, ESSALUD, Lima, Perú ${ }^{34}$; Centro Nacional de Enfermedades Reumáticas, Hospital Universitario de Caracas, Caracas, Venezuela ${ }^{35}$;Hospital Central de San Cristóbal, San Cristóbal, Venezuela ${ }^{36}$. 
Palabras clave:

antimaláricos, lupus eritematoso sistémico, daño acumulado,

\section{R E S U M E N}

Objetivos: Estimar el efecto de los antimaláricos (AM) sobre los diferentes dominios del índice de daño SLICC (SDI).

Métodos: Se estudiaron pacientes con diagnóstico clínico reciente ( $\leq 2$ años) de lupus eritematoso sistémico (LES) de la cohorte GLADEL. Variable de estudio: aumento en los dominios del SDI desde el ingreso a la cohorte. Variables independientes: características sociodemográficas, clínicas, laboratorio y tratamientos. El efecto de los AM, como variable dependiente del tiempo, sobre los dominios más frecuentes del SDI (ajustado por factores de confusión) fue examinado con un modelo de regresión de Cox multivariado.

Resultados: De 1466 pacientes estudiados, 1049 (72\%) recibieron AM con un tiempo medio de exposición de 30 meses (Q1-Q3: 11-57) y 665 pacientes (45\%) presentaron daño durante un seguimiento medio de 24 meses (Q1-Q3: 8-55); 301 eventos fueron cutáneos, 208 renales, 149 neuropsiquiátricos, 98 musculoesqueléticos, 88 cardiovasculares y 230 otros. Después de ajustar por factores de confusión, el uso de AM se asoció a un menor riesgo de daño renal (HR 0,652; IC 95\%: 0,472-0,901) y en el límite de la significancia estadística (HR 0,701, IC 95\%: 0,481-1,024) para el dominio neuropsiquiátrico.

Conclusión: En GLADEL, el uso de AM se asoció independientemente a un menor riesgo de daño acumulado renal.

\section{A B S T R A C T}

Objective: To assess the effects of antimalarials (AM) over the items of the SLICC Damage Index (SDI).

Methods: Patients with recent ( $\leq 2$ years) diagnosis of systemic lupus erythematosus (SLE) from the GLADEL cohort were studied. End-point: increase in items SDI since cohort entry. Independent variables (socio-demographic, clinical, laboratory and treatment) were included. The effect of AM as a time dependent variable on most frequent SDI items (adjusting for potential confounders) was examined with a multivariable Cox regression model.

Results: Of the 1466 patients included in this analysis, 1049 (72\%) received AM with a median exposure time of 30 months (Q1-Q3: 11-57). Damage occurred in 665 (45\%) patients during a median follow-up time of 24 months (Q1-Q3: 8-55). There were 301 integument, 208 renal, 149 neuropsychiatric, 98 musculoskeletal, 88 cardiovascular and 230 others less frequently represented damages. After adjusting for potential confounders at any time during follow-up, a lower risk of renal damage (HR 0.652; 95\% CI: 0.472-0.901) and borderline for neuropsychiatric damage (HR 0.701, 95\% CI: 0.481-1.024) was found.

Conclusion: In the GLADEL cohort, after adjustment for possible confounding factors, AM were independently associated with a reduced risk of renal damage accrual.

Correspondencia

E-mail: gponsestel@yahoo.com

\section{Introducción}

El lupus eritematoso sistémico (LES) es una enfermedad autoinmune compleja, de etiología desconocida y multifactorial. El espectro de las manifestaciones clínicas incluye desde formas leves hasta el compromiso severo ${ }^{1}$. A pesar de una mayor accesibilidad en la atención médica especializada y diagnóstico precoz, así como de la mejoría en los tratamientos, los pacientes con lupus presentan una elevada morbimortalidad ${ }^{2}$ dependiente de la actividad, comorbilidades y daño producido durante la evolución de la enfermedad. El daño acumulado, en particular, no se debe solo a la enfermedad en sí misma, sino que también suele asociarse a los tratamientos utilizados para el control de la actividad lúpica, siendo el más común ejemplo la aparición de daño secundario al uso de glucocorticoides (GCs) ${ }^{3}$. Por el contrario, el uso de antimaláricos (AM) ha demostrado un efecto protector sobre la aparición de daño en general ${ }^{4-10}$ y en particular, sobre los dominios cutáneos, renales y neuropsiquiátricos ${ }^{4,5,11}$. El objetivo del presente estudio fue evaluar si el uso de AM retrasaría la aparición de daño sobre 
los diferentes dominios que componen el índice de daño SLICC (SDI).

\section{Material y método}

Se estudiaron pacientes con diagnóstico reciente de LES ( $\leq 2$ años) pertenecientes a la cohorte del Grupo Latino Americano De Estudio del Lupus (GLADEL). GLADEL es una cohorte de incepción, multinacional y multiétnica, formada en el año 1997 con el objetivo de estudiar las características sociodemográficas, clínicas y terapéuticas de los pacientes con lupus de Latinoamérica ${ }^{12}$. Esta cohorte está formada por 34 centros de 9 países de Latinoamérica. El diagnóstico se realizó en base a las características clínicas y de laboratorio presentes según el juicio experto de los investigadores. No se consideró obligatorio el cumplimiento de 4 o más criterios del Colegio Americano de Reumatología (ACR) $1982^{12}$. Sin embargo, durante la evolución, el $97 \%$ de los pacientes de GLADEL cumplieron con dichos criterios de clasificación $^{13}$. El objetivo primario o variable dependiente del estudio fue la aparición luego del ingreso en la cohorte, de alguno de los 12 dominios que componen el SDI ${ }^{14,15}$. Como variables independientes, se evaluaron características sociodemográficas, manifestaciones clínicas, laboratorio y tratamiento. El "uso de AM" fue definido como una variable dependiente del tiempo, valorándose el tiempo de exposición al fármaco antes de la aparición del evento.

\section{Análisis estadístico}

El efecto de los AM sobre los diferentes dominios del índice SDI, fue examinado a través de un modelo de regresión de Cox multivariado, ajustado por variables de confusión relacionadas con la aparición de daño cutáneo, renal, neuropsiquiátrico, musculoesquelético y cardiovascular. Debido al bajo número de eventos oculares, pulmonares, sistema vascular periférico, gastrointestinales, falla gonadal prematura, diabetes y tumores malignos, el uso de AM sobre estos dominios del SDI no pudo ser valorado. Los resultados se presentan como Hazard Ratio (HR) y su respectivo intervalo de confianza del 95\% (IC 95\%). El nivel de significancia estadística fue $\leq 0,05$. Se utilizó, para el análisis, el paquete estadístico SAS v9.4 (SAS Institute Inc, Cary, NC).

\section{Aspectos éticos}

En la cohorte GLADEL, todos los investigadores siguieron las regulaciones locales establecidas por los diferentes Comités de Ética de cada uno de los centros participantes. En todo momento se ha velado por la protección de los datos personales de los pacientes incluidos en el estudio.

\section{Resultados}

Un total de 1466 pacientes fueron incluidos en el análisis; de ellos, 480 (32,7\%) recibieron tratamiento con AM al ingreso a la cohorte y 1049 (72\%) lo hicieron durante el seguimiento. La mediana de seguimiento fue de 52 meses (Q1-Q3: 2570) y la mediana de exposición a los AM fue de 30 meses
Tabla 1. Modelo de regresión de Cox con la variable uso de AM como variable tiempo dependiente.

\begin{tabular}{|c|c|c|c|c|}
\hline \multirow[t]{2}{*}{ Variables } & \multicolumn{2}{|c|}{ Modelo no ajustado } & \multicolumn{2}{|c|}{ Modelo ajustado } \\
\hline & $\mathrm{HR}^{1}$ (IC 95\%) & $\mathrm{P}$ & $\mathrm{HR}^{1}$ (IC 95\%) & $\mathrm{p}$ \\
\hline Daño cutáneo & $\begin{array}{c}0,987 \\
(0,763-1,277)\end{array}$ & 0,9223 & $\begin{array}{c}0,971 \\
(0,734-1,286)^{2}\end{array}$ & 0,8381 \\
\hline Daño $r$ & $\begin{array}{c}0,516 \\
(0,385-0,692) \\
\end{array}$ & $<0,0001$ & $\begin{array}{c}0,652 \\
(0,472-0,901)^{3}\end{array}$ & 0,0094 \\
\hline $\begin{array}{l}\text { Daño } \\
\text { neuropsiquiátrico }\end{array}$ & $\begin{array}{c}0,651 \\
(0,458-0,925)\end{array}$ & 0,0167 & $\begin{array}{c}0,701 \\
(0,481-1,024)^{4}\end{array}$ & 0,0660 \\
\hline $\begin{array}{l}\text { Daño } \\
\text { musculoesquelético }\end{array}$ & $\begin{array}{c}0,838 \\
(0,524-1,340)\end{array}$ & 0,4612 & $\begin{array}{c}0,909 \\
(0,561-1,473)^{5}\end{array}$ & 0,6977 \\
\hline $\begin{array}{l}\text { Daño } \\
\text { cardiovascular }\end{array}$ & $\begin{array}{c}0,562 \\
(0,357-0,886)\end{array}$ & 0,0130 & $\begin{array}{c}0,690 \\
(0,430-1,107)^{6}\end{array}$ & 0,1240 \\
\hline
\end{tabular}

${ }^{1} \mathrm{HR}$ sobre el uso de AM en el mes previo versus el no uso en el mes previo. ${ }^{2}$ Ajustado por las siguientes variables: daño cutáneo al ingreso a la cohorte, hipertensión, rash malar, rash discoide, proteinuria/cilindruria, manifestaciones hematológicas, pulsos de glucocorticoides y SLEDAI al ingreso a la cohorte. ${ }^{3}$ Ajustado por las siguientes variables: daño renal al ingreso a la cohorte, edad al diagnóstico, nivel socioeconómico, hipertensión, proteinuria/cilindruria, uso de inmunosupresores y SLEDAI al ingreso a la cohorte. ${ }^{4} \mathrm{Ajustado}$ por las siguientes variables: daño neurológico al ingreso a la cohorte, pulsos de glucocorticoides y SLEDAI al ingreso a la cohorte. ${ }^{5}$ Ajustado por las siguientes variables: daño musculoesquelético al ingreso a la cohorte, hipertensión, género, rash discoide, úlceras orales, artritis, manifestaciones neurológicas y uso de glucocorticoides al ingreso a la cohorte. ${ }^{6} \mathrm{Ajustado}$ por las siguientes variables: daño cardiovascular al ingreso a la cohorte, duración de la enfermedad, hipertensión y serositis.

(Q1-Q3: 11-57 meses). Un total de 665 (45\%) pacientes desarrollaron daño durante una mediana de seguimiento de 24 meses (Q1-Q3: 8-55). La frecuencia de daño por dominio fue: 301 (20,5\%) eventos cutáneos, 208 (14,2\%) renales, 149 $(10,2 \%)$ neuropsiquiátricos, $98(6,7 \%)$ musculoesqueléticos, $88(6,0 \%)$ cardiovasculares, $65(4,4 \%)$ oculares, $43(2,9 \%)$ pulmonares, $42(2,9 \%)$ sistema vascular periférico, $33(2,3 \%)$ gastrointestinales, $22(1,5 \%)$ falla gonadal prematura, 16 $(1,1 \%)$ diabetes y $9(0,6 \%)$ tumores malignos. En la Tabla 1 se puede observar el efecto de los AM sobre los diferentes dominios del SDI. En los modelos que no fueron ajustados por variables de confusión se puede observar que el uso de AM se asoció con un menor riesgo de daño renal (HR 0,516; IC 95\%: 0,385-0,692), neuropsiquiátrico (HR 0,651; IC 95\%: 0,458-0,925) y cardiovascular (HR 0,562; IC 95\%: 0,357-0,886). Sin embrago, después de ajustar por los posibles factores de confusión, en cualquier momento durante el seguimiento, los pacientes que fueron tratados con AM presentaron menor riesgo de daño acumulado en los dominios renal y neuropsiquiátrico (35\% y un 30\%, respectivamente) (aunque este último en el límite de la significancia estadística) en comparación con aquellos pacientes que no recibieron tratamiento con AM (HR ajustado 0,652; IC 95\%: 0,472-0,901 y HR 0,701, IC 95\%: 0,481-1,024). Tal efecto protector no fue evidente para los dominios cutáneo, musculoesquelético y cardiovascular.

\section{Discusión}

Los AM son unos de los fármacos más antiguamente utilizados en el tratamiento del LES. Su eficacia, bajo perfil 
de eventos adversos y el bajo costo los han transformado en parte del tratamiento estándar de los paciente con lupus ${ }^{16,17}$. En el presente estudio, y luego de ajustar por posibles variables de confusión, hemos podido demostrar por primera vez, en pacientes Latinoamericanos, que el uso de AM está asociado, de forma independiente, con una reducción del daño acumulado a nivel renal y de manera limítrofe a la significancia estadística con el daño neuropsiquiátrico. Estos hallazgos, y otros publicados sobre el uso de AM en pacientes Latinoamericanos y de otras cohortes, afirman la necesidad de utilizar estos fármacos como base del tratamiento en todos los pacientes con lupus, en caso de no existir contraindicaciones ${ }^{18-20}$.

El daño, generado por la enfermedad crónica y/o los tratamientos utilizados, es una de las complicaciones más temidas del lupus. Datos de la cohorte LUMINA (LUpus in MInorities, NAture versus nurture), han demostrado que el tratamiento con AM disminuye la posibilidad de desarrollar daño en pacientes que no presentaban esta complicación al ingreso a la cohorte, así como tampoco la aparición de un evento de daño nuevo ${ }^{21}$. Estos datos han sido corroborados en pacientes Mestizos, Caucásicos y Afro-Latinoamericanos de la cohorte GLADEL ${ }^{10}$, donde los AM demostraron una reducción del $25 \%$ en el desarrollo de un aumento del índice de daño acumulado.

Los AM también han demostrado un efecto protector sobre el compromiso renal en el lupus. Kasitanon y cols. ${ }^{22}$ demostraron que los pacientes con nefritis lúpica membranosa tratados con mofetil micofenolato fueron más propensos a lograr remisión después de 1 año de tratamiento, cuando concomitantemente fueron tratados con hidroxicloroquina. Barber y cols. ${ }^{23}$ establecieron que la remisión renal sostenida es más probable de ser lograda en pacientes tratados con hidroxicloroquina $(93,8$ vs $52,6 \%$, $\mathrm{p}=0,010)$. Estudios realizados en la cohorte GLADEL ${ }^{18,19}$ demostraron que el uso de AM no se asocia solamente a una menor probabilidad de presentar cilindruria y proteinuria sino que además retrasaría la aparición de dichas manifestaciones. En términos de daño renal, Siso y cols. ${ }^{24}$ estudiaron 206 pacientes con nefritis lúpica confirmada por biopsia renal, y demostraron que los pacientes tratados con $\mathrm{AM}$, antes de que ocurriera la nefritis, tenían una probabilidad 9\% menor de desarrollar enfermedad renal terminal que aquellos que nunca fueron tratados. Finalmente, PonsEstel y cols. ${ }^{5}$ demostraron, en 635 de la cohorte LUMINA, que el uso de AM se asoció con un retraso en la aparición de daño renal en aquellos pacientes con nefritis activa. Es importante remarcar, que solo los datos de las cohortes LUMINA y GLADEL fueron ajustados por posibles factores confundidores.

En relación al daño neuropsiquiátrico, González y cols. ${ }^{11}$ evaluaron el tiempo necesario para la ocurrencia de daño neuropsiquiátrico en pacientes pertenecientes a la cohorte de LUMINA. Utilizando análisis de tiempo al evento, dichos autores demostraron que el uso de hidroxicloroquina retardaba en un $42 \%$ la aparición de daño. Finalmente, García y cols. ${ }^{25}$ demostraron que el uso de AM disminuye hasta un $42 \%$ la aparición de enfermedad cardíaca primaria, definida como la presencia de una o más de las siguientes manifestaciones: pericarditis, miocarditis, endocarditis, arritmias y/o valvulopatía, anormalidades resultantes de mecanismos autoinmune. Estos datos son concordantes con nuestros hallazgos, que probablemente no mostraron significancia estadística debido al bajo número de eventos neurológicos y cardiovasculares asociado a un seguimiento menor de 5 años en esta cohorte de incepción.

\section{Conclusión}

Los AM no solo disminuyen el riesgo de nuevos brotes, sino que también previenen la aparición de daño en diferentes órganos, todo lo cual justifica una mayor sobrevida de los pacientes con lupus. Con los datos expuestos, enfatizamos la importancia del tratamiento con AM, en forma temprana y por tiempo prolongado, de todos los pacientes con lupus, independientemente de la severidad de sus manifestaciones clínicas.

\section{BIBLIOGRAFÍA}

1. Boumpas DT, Austin HA, 3rd, Fessler BJ, Balow JE, Klippel JH, Lockshin MD. Systemic lupus erythematosus: emerging concepts. Part 1: Renal, neuropsychiatric, cardiovascular, pulmonary, and hematologic disease. Annals of internal medicine. 1995;122(12):940-50.

2. Boumpas DT, Fessler BJ, Austin HA, 3rd, Balow JE, Klippel $\mathrm{JH}$, Lockshin MD. Systemic lupus erythematosus: emerging concepts. Part 2: Dermatologic and joint disease, the antiphospholipid antibody syndrome, pregnancy and hormonal therapy, morbidity and mortality, and pathogenesis. Annals of internal medicine. 1995;123(1):4253.

3. Fessler BJ, Boumpas DT. Severe major organ involvement in systemic lupus erythematosus. Diagnosis and management. Rheumatic diseases clinics of North America. 1995;21(1):81-98.

4. Pons-Estel GJ, Alarcon GS, Gonzalez LA, Zhang J, Vila LM, Reveille JD, et al. Possible protective effect of hydroxychloroquine on delaying the occurrence of integument damage in lupus: LXXI, data from a multiethnic cohort. Arthritis care \& research. 2010;62(3):393-400.

5. Pons-Estel GJ, Alarcon GS, McGwin G, Jr., Danila MI, Zhang J, Bastian HM, et al. Protective effect of hydroxychloroquine on renal damage in patients with lupus nephritis: LXV, data from a multiethnic US cohort. Arthritis and rheumatism. 2009;61(6):830-9.

6. Pons-Estel GJ, Gonzalez LA, Zhang J, Burgos PI, Reveille JD, Vila LM, et al. Predictors of cardiovascular damage in patients with systemic lupus erythematosus: data from LUMINA (LXVIII), a multiethnic US cohort. Rheumatology. 2009;48(7):817-22.

7. Alarcon GS, McGwin G, Bertoli AM, Fessler BJ, Calvo-Alen J, Bastian HM, et al. Effect of hydroxychloroquine on the survival of patients with systemic lupus erythematosus: data from LUMINA, a multiethnic US cohort (LUMINA L). Annals of the rheumatic diseases. 2007;66(9):1168-72.

8. Akhavan PS, Su J, Lou W, Gladman DD, Urowitz MB, Fortin PR. The early protective effect of hydroxychloroquine on the risk of cumulative damage in patients with systemic 
lupus erythematosus. The Journal of rheumatology. 2013;40(6):831-41.

9. Molad Y, Gorshtein A, Wysenbeek AJ, Guedj D, Majadla R, Weinberger A, et al. Protective effect of hydroxychloroquine in systemic lupus erythematosus. Prospective long-term study of an Israeli cohort. Lupus. 2002;11(6):356-61.

10. Pons-Estel GWD, Ugarte-Gil M, et al 192 Protective effect of, erythematosus aotrodaisl, 10.1136/lupus-2017-000215.192 LSMd.

11. Gonzalez LA, Pons-Estel GJ, Zhang J, Vila LM, Reveille JD, Alarcon GS, et al. Time to neuropsychiatric damage occurrence in LUMINA (LXVI): a multi-ethnic lupus cohort. Lupus. 2009;18(9):822-30.

12. Tan EM, Cohen AS, Fries JF, Masi AT, McShane DJ, Rothfield NF, et al. The 1982 revised criteria for the classification of systemic lupus erythematosus. Arthritis and rheumatism. 1982;25(11):1271-7.

13. Pons-Estel GJ, Wojdyla D, McGwin G, Jr., Magder LS, Petri MA, Pons-Estel BA, et al. The American College of Rheumatology and the Systemic Lupus International Collaborating Clinics classification criteria for systemic lupus erythematosus in two multiethnic cohorts: a commentary. Lupus. 2014;23(1):3-9.

14. Gladman D, Urowitz M, Fortin P, Isenberg D, Goldsmith C, Gordon C, et al. Systemic Lupus International Collaborating Clinics conference on assessment of lupus flare and quality of life measures in SLE. Systemic Lupus International Collaborating Clinics Group. The Journal of rheumatology. 1996;23(11):1953-5.

15. Gladman DD, Urowitz MB, Goldsmith $\mathrm{CH}$, Fortin P, Ginzler E, Gordon C, et al. The reliability of the Systemic Lupus International Collaborating Clinics/American College of Rheumatology Damage Index in patients with systemic lupus erythematosus. Arthritis and rheumatism. 1997;40(5):809-13.

16. Dall'era M, Chakravarty EF. Treatment of mild, moderate, and severe lupus erythematosus: focus on new therapies. Curr Rheumatol Rep. 2011;13(4):308-16.

17. Ruiz-Irastorza G, Ramos-Casals M, Brito-Zeron $P$, Khamashta MA. Clinical efficacy and side effects of antimalarials in systemic lupus erythematosus: a systematic review. Annals of the rheumatic diseases. 2010;69(1):20-8.

18. Pons-Estel GJ, Alarcon GS, Burgos PI, Hachuel L, Boggio G, Wojdyla D, et al. Mestizos with systemic lupus erythematosus develop renal disease early while antimalarials retard its appearance: data from a Latin American cohort. Lupus. 2013;22(9):899-907.

19. Pons-Estel GJ, Alarcon GS, Hachuel L, Boggio G, Wojdyla D, Pascual-Ramos V, et al. Anti-malarials exert a protective effect while Mestizo patients are at increased risk of developing SLE renal disease: data from a Latin-American cohort. Rheumatology. 2012;51(7):1293-8.

20. Shinjo SK, Bonfa E, Wojdyla D, Borba EF, Ramirez LA, Scherbarth HR, et al. Antimalarial treatment may have a time-dependent effect on lupus survival: data from a multinational Latin American inception cohort. Arthritis and rheumatism. 2010;62(3):855-62.

21. Fessler BJ, Alarcon GS, McGwin G,Jr., Roseman J, Bastian HM, Friedman AW, et al. Systemic lupus erythematosus in three ethnic groups: XVI. Association of hydroxychloroquine use with reduced risk of damage accrual. Arthritis and rheumatism. 2005;52(5):1473-80.

22. Kasitanon N, Fine DM, Haas M, Magder LS, Petri M. Hydroxychloroquine use predicts complete renal remission within 12 months among patients treated with mycophenolate mofetil therapy for membranous lupus nephritis. Lupus. 2006;15(6):366-70.

23. Barber CE, Geldenhuys L, Hanly JG. Sustained remission of lupus nephritis. Lupus. 2006;15(2):94-101.

24. Siso A, Ramos-Casals M, Bove A, Brito-Zeron P, Soria N, Munoz S, et al. Previous antimalarial therapy in patients diagnosed with lupus nephritis: influence on outcomes and survival. Lupus. 2008;17(4):281-8.

25. Garcia MA, Alarcon GS, Boggio G, Hachuel L, Marcos AI, Marcos JC, et al. Primary cardiac disease in systemic lupus erythematosus patients: protective and risk factors--data from a multi-ethnic Latin American cohort. Rheumatology. 2014;53(8):1431-8. 